\title{
Constraints on neutrino masses from Planck and Galaxy clustering data
}

\author{
Elena Giusarma, ${ }^{1}$ Roland de Putter, ${ }^{2}$ Shirley Ho,${ }^{3}$ and Olga Mena ${ }^{1}$ \\ ${ }^{1}$ IFIC, Universidad de Valencia-CSIC, 46071 Valencia, Spain \\ ${ }^{2}$ Jet Propulsion Laboratory, California Institute of Technology, Pasadena, California 91109, USA, \\ and California Institute of Technology, Pasadena, California 91125, USA \\ ${ }^{3}$ Department of Physics, McWilliams Center for Cosmology, Carnegie Mellon University, \\ 5000 Forbes Avenue, Pittsburgh, Pennsylvania 15213, USA \\ (Received 26 June 2013; published 10 September 2013)
}

\begin{abstract}
We present here bounds on neutrino masses from the combination of recent Planck cosmic microwave background $(\mathrm{CMB})$ measurements and galaxy clustering information from the Baryon Oscillation Spectroscopic Survey, part of the Sloan Digital Sky Survey-III. We use the full shape of either the photometric angular clustering (Data Release 8) or the 3D spectroscopic clustering (Data Release 9) power spectrum in different cosmological scenarios. In the $\Lambda$ CDM scenario, spectroscopic galaxy clustering measurements improve significantly the existing neutrino mass bounds from Planck data. We find $\sum m_{\nu}<0.39 \mathrm{eV}$ at $95 \%$ confidence level for the combination of the 3D power spectrum with Planck CMB data (wi lensing included) and Wilkinson Microwave Anisoptropy Probe 9-year polarization measurements. Therefore, robust neutrino mass constraints can be obtained without the addition of the prior on the Hubble constant from Hubble Space Telescope. In extended cosmological scenarios with a dark energy fluid or with nonflat geometries, galaxy clustering measurements are essential to pin down the neutrino mass bounds, providing in the majority of cases better results than those obtained from the associated measurement of the baryon acoustic oscillation scale only. In the presence of a freely varying (constant) dark energy equation of state, we find $\sum m_{\nu}<0.49 \mathrm{eV}$ at $95 \%$ confidence level for the combination of the 3D power spectrum with Planck CMB data (with lensing included) and Wilkinson Microwave Anisoptropy Probe 9-year polarization measurements. This same data combination in nonflat geometries provides the neutrino mass bound $\sum m_{\nu}<0.35 \mathrm{eV}$ at $95 \%$ confidence level.
\end{abstract}

\section{INTRODUCTION}

Massive neutrinos leave distinct imprints in the different cosmological data sets. Concerning cosmic microwave background $(\mathrm{CMB})$ anisotropies, the primary effect of neutrino masses is via the Early Integrated Sachs Wolfe effect. The transition from the relativistic to the nonrelativistic neutrino regime will affect the decays of the gravitational potentials at the decoupling period, leading to a non-negligible signature around the first peak. This has been, traditionally, the most relevant signature from neutrino masses on the CMB [1]. However, recent neutrino mass bounds from Planck data [2] seem to be driven by the massive neutrino signature on gravitational lensing. A nonzero value of the neutrino mass will induce a higher expansion rate which will suppress the clustering on scales smaller than the horizon while neutrinos turn nonrelativistic [3]. Regarding large scale structure, due to the large neutrino velocity dispersion, the nonrelativistic neutrino overdensities will only cluster at wavelengths larger than their free streaming scale. Consequently, the growth of matter density perturbations is reduced and the matter power spectrum is suppressed at small scales; see Ref. [4] and references therein. Therefore, cosmological data provide a unique tool to test the neutrino masses; see Refs. [5-13] for neutrino mass bounds before Planck CMB data release.
The limits from the Planck satellite, including lensing as well as low- $\ell$ polarization measurements from WMAP 9-year data [14] (WP) are $\sum m_{\nu}<1.11 \mathrm{eV}$ at 95\% C.L. The addition of a prior on the Hubble constant $H_{0}$ from the Hubble Space Telescope [15] improves the constraint in a very significant way, $\sum m_{\nu}<0.21 \mathrm{eV}$ at $95 \%$ C.L. This is due to the strong degeneracy between $H_{0}$ and $\sum m_{\nu}$ : if the sum of the neutrino masses is increased, the change induced in the distance to last scattering can be compensated by lowering $H_{0}$ [8]. However, Planck and Hubble Space Telescope (HST) measurements of the Hubble constant $H_{0}$ show a $2.5 \sigma$ tension and therefore, it is fortunate that data sets other than the HST prior may help in pinning down the bound on neutrino mass from CMB data alone.

Baryon acoustic oscillation (BAO) data, as measured by the Sloan Digital Sky Survey (SDSS) Data Release 7 [16,17], the WiggleZ survey [18], the Baryon Oscillation Spectroscopic Survey (BOSS) SDSS-III Data Release 9 [19] and 6dF [20], also significantly improve the constraints, leading to $\sum m_{\nu}<0.26 \mathrm{eV}$ at $95 \%$ C.L. when combined with Planck (with lensing) and WP data. However, in nonminimal scenarios with a curvature or with a more general dark energy component these constraints are notably degraded and geometrical BAO information from galaxy clustering may not be as powerful as shape measurements of the matter power spectrum. Previous works $[6,8]$ have 
noticed the advantages of using full power spectrum measurements in extended cosmological scenarios due to their ability of removing degeneracies.

Here we combine recent Planck data with galaxy power spectrum measurements from the BOSS experiment [21], one of the four surveys of the Sloan Digital Sky Survey III, SDSS-III [22]. We consider first the 2D angular power spectrum measurements [23] from the CMASS sample [24] of luminous galaxies of SDSS Data Release 8 (DR8) [25]. We then explore as well the neutrino mass constraints from the full 3D power spectrum shape of SDSS Data Release 9 (DR9) [26]. While DR8 contains the full photometric CMASS sample, DR9 provides the galaxy spectra of CMASS galaxies, the largest publicly available set of galaxy spectra to date.

The authors of Ref. [7], in the context of a $\Lambda \mathrm{CDM}$ model, found $\sum m_{\nu}<0.36 \mathrm{eV}\left(\sum m_{\nu}<0.26 \mathrm{eV}\right)$ at 95\% C.L. with (without) shot noiselike parameters when combining WMAP 7-year data with DR8 2D angular power spectrum measurements plus a HST prior on $H_{0}$. Exploiting DR9 3D power spectrum measurements Ref. [9] quotes the bound $\sum m_{\nu}<0.34 \mathrm{eV}$ at $95 \%$ C.L. after combining with WMAP7, supernova data and additional BAO measurements within a $\Lambda$ CDM model.

We shall update here the constraints quoted above, quantifying the benefits from the improved CMB Planck data. Our neutrino mass constraints are presented in different fiducial cosmologies, namely, nonflat and dynamical dark energy cosmologies. We also show the impact on our constraints of the underlying galaxy power spectrum, adopting different models to describe galaxy clustering.

The structure of the paper is as follows. In Sec. II we describe the parameters used in the analysis. Planck CMB and galaxy clustering data, plus galaxy clustering modeling are described in Sec. III. Section IV contains our results, and we draw our conclusions in Sec. V.

\section{COSMOLOGICAL PARAMETERS}

The standard, three massive neutrino scenario we explore here is described by the following set of parameters:

$$
\left\{\omega_{b}, \omega_{c}, \Theta_{s}, \tau, n_{s}, \log \left[10^{10} A_{s}\right], \sum m_{\nu}\right\},
$$

$\omega_{b} \equiv \Omega_{b} h^{2}$ and $\omega_{c} \equiv \Omega_{c} h^{2}$ being the physical baryon and cold dark matter energy densities, $\Theta_{s}$ the ratio between the sound horizon and the angular diameter distance at decoupling, $\tau$ is the reionization optical depth, $n_{s}$ the scalar spectral index, $A_{s}$ the amplitude of the primordial spectrum and $\sum m_{\nu}$ the sum of the masses of the three active neutrinos in $\mathrm{eV}$. We assume a degenerate neutrino mass spectrum in the following. The former scenario is enlarged with $w$ and $\Omega_{k}$ in the case of extended models. Table I specifies the priors considered on the different cosmological parameters. For our numerical analyses, we have used the Boltzmann CAMB code [27] and extracted cosmological
TABLE I. Uniform priors for the cosmological parameters considered here.

\begin{tabular}{lc}
\hline \hline Parameter & Prior \\
\hline$\Omega_{b} h^{2}$ & $0.005 \rightarrow 0.1$ \\
$\Omega_{c} h^{2}$ & $0.01 \rightarrow 0.99$ \\
$\Theta_{s}$ & $0.5 \rightarrow 10$ \\
$\tau$ & $0.01 \rightarrow 0.8$ \\
$n_{s}$ & $0.9 \rightarrow 1.1$ \\
$\ln \left(10^{10} A_{s}\right)$ & $2.7 \rightarrow 4$ \\
$\sum_{\Omega_{\nu}} m_{\nu}(\mathrm{eV})$ & $0.06 \rightarrow 3$ \\
$w$ & $-0.3 \rightarrow 0.3$ \\
\hline \hline
\end{tabular}

parameters from current data using a Monte Carlo Markov chain (MCMC) analysis based on the publicly available MCMC package COSMOMC [28].

\section{CMB AND GALAXY CLUSTERING MEASUREMENTS}

\section{A. Planck}

We consider the high- $\ell$ TT likelihood, including measurements up to a maximum multipole number of $\ell_{\max }=$ 2500 , combined with the low- $\ell$ TT likelihood, including measurements up to $\ell=49$ and the low- $\ell$ WMAP TE,EE, $\mathrm{BB}$ likelihood including multipoles up to $\ell=23$. We include the lensing likelihood in all our Monte Carlo analyses. We refer to this data set as the PLANCK data set.

We also consider the effect of a Gaussian prior on the Hubble constant $H_{0}=73.8 \pm 2.4 \mathrm{~km} / \mathrm{s} / \mathrm{Mpc}$, accordingly with the measurements from the Hubble Space Telescope [15]. We refer to this prior as HST.

We fix the helium abundance to $Y_{p}=0.24$ and the lensing spectrum normalization to $A_{L}=1$. We marginalize over all foregrounds parameters as described in [2].

\section{B. DR8 angular power spectrum \\ 1. DR8 data}

We exploit the stellar mass-limited DR8 CMASS sample of luminous galaxies, detailed in [24], divided into four photometric redshift bins, $z=0.45-0.5-0.55-0.6-0.65$. The photometric redshift error lies within the range $\sigma_{z}(z)=0.04-0.06$, increasing from low to high redshift, see Refs. [23,29]. The calculation of the angular power spectrum for each bin is described in detail in Ref. [23]. The expectation value of the power spectrum is a convolution of the true power spectrum with a window function, see [30] for examples on these window functions. When fitting the data to the underlying theoretical model, we always apply these window functions to the theoretical power spectra before calculating the likelihood relative to the data. To avoid large systematic uncertainties [23,29] we do not consider bands with $\ell<30$ in our analysis. 


\section{DR8 clustering model}

In order to describe the theoretical angular power spectrum, we follow here the simple linear scale independent bias model described in Ref. [7], characterized by four free bias parameters $b_{i}$ (i.e. one per each redshift bin). In addition to these bias parameters, we also consider shot noiselike parameters $a_{i}$ :

$$
\begin{aligned}
C_{\ell}^{(i i)}= & b_{i}^{2} \frac{2}{\pi} \int k^{2} d k P^{\mathrm{m}}(k, z=0) \times\left(\Delta_{\ell}^{(i)}(k)\right. \\
& \left.+\Delta_{\ell}^{\mathrm{RSD},(i)}(k)\right)^{2}+a_{i},
\end{aligned}
$$

where the $a_{i}$ parameters mimic the effects of a scaledependent galaxy bias as well as the effect of potential insufficient shot noise subtraction. Table II denotes the priors adopted on the bias and shot noise parameters in each of the four redshift bins exploited here. The neutrino mass bounds presented in the next section will be derived by default including the shot noise parameters $a_{i}$ in the next section, although we shall mention on some cases the bounds without shot noise. In Eq. (2), $P^{\mathrm{m}}(k, z=0)$ is the matter power spectrum at redshift zero after applying the HaloFit prescription [31,32] to account for nonlinear effects ${ }^{1}$ and

$$
\Delta_{\ell}^{(i)}(k)=\int d z g_{i}(z) T(k, z) j_{\ell}(k d(z)) .
$$

Here, $g_{i}(z)$ is the normalized redshift distribution of galaxies in bin $i, j_{\ell}$ is the spherical Bessel function, $d(z)$ is the comoving distance to redshift $z$ and $T(k, z)$ the matter transfer function relative to redshift zero. The contribution due to redshift space distortions is

$$
\begin{aligned}
\Delta_{l}^{\mathrm{RSD},(i)}(k)= & \beta_{i} \int d z g_{i}(z) T(k, z) \\
& \times\left[\frac{\left(2 l^{2}+2 l-1\right)}{(2 l+3)(2 l-1)} j_{l}(k d(z))\right. \\
& -\frac{l(l-1)}{(2 l-1)(2 l+1)} j_{l-2}(k d(z)) \\
& \left.-\frac{(l+1)(l+2)}{(2 l+1)(2 l+3)} j_{l+2}(k d(z))\right],
\end{aligned}
$$

where $\beta_{i}(z)=f(z) / b_{i}$ is the redshift distortion parameter and

\footnotetext{
${ }^{1}$ Although the revisited version of the HaloFit model [32] accounts for a constant, $w \neq-1$ dark energy equation of state, it is restricted to flat models. In principle, a linear interpolation of the fitting functions to $\mathrm{N}$-body simulations could account for not flat models [33]. In practice, since these fitting functions have been shown to have a 5\%-10\% discrepancy with simulations even in the simplest case of a flat $\Lambda$ CDM scheme, (see e.g. Ref. [34] and references therein), we neglect here the extra corrections in the HaloFit description in not flat cosmologies.
}

TABLE II. Uniform priors for the DR8 bias and shot noise parameters in each of the four photometric redshift bins $z=0.45-0.5-0.55-0.6-0.65$ used in the DR8 clustering data analyses.

\begin{tabular}{lc}
\hline \hline DR8 parameters & Prior \\
\hline$b_{i}$ & $0.5 \rightarrow 5$ \\
$a_{i}$ & $-5 \rightarrow 12$ \\
\hline \hline
\end{tabular}

$$
f(z) \equiv \frac{d \ln D(z)}{d \ln a}
$$

is the growth factor [with $D(z)$ the linear growth function]. When massive neutrinos are an additional ingredient in the Universe's mass energy density, the growth function is scale dependent. Following Ref. [7], we shall ignore the scale dependent growth in $\beta(z)$ since it is a small $(\ll 10 \%)$ correction to the already small effect of redshift space distortions.

As previously stated, $\ell>30$ in our data analyses. We consider $\ell_{\max }=200$, value which ensures the suppression of the uncertainties from nonlinear corrections to the modeled angular power spectra [7]. For the likelihood function, we use 17 data points per redshift slice.

\section{DR9 power spectrum \\ 1. DR9 data}

Here we use the DR9 CMASS sample of galaxies [26] which contains 246283 massive galaxies covering $3275 \mathrm{deg}^{2}$ with redshifts $0.43<z<0.7$ (being the effective redshift $z_{\text {eff }}=0.57$ ). The measured galaxy power spectrum $P_{\text {meas }}(k)$ is the one used in Refs. [9,19,35-40], which is obtained using the standard Fourier technique [41]; see [42] for details. This galaxy power spectrum was the one used to fit the baryon acoustic oscillations [19].

On large scales, we are affected by systematic effects from stars or seeing of the survey. On small scales, we are affected by observational effects such as redshift failures and fiber collisions. A conservative approach has been provided by Refs. [39,40], which adds an extra free parameter in the measured power spectrum,

$P_{\text {meas }}(k)=P_{\text {meas, } \mathrm{w}}(k)-S\left[P_{\text {meas, nw }}(k)-P_{\text {meas, } \mathrm{w}}(k)\right]$,

where $P_{\text {meas,w }}(k)$ refers to the measured power spectrum after applying the weights for stellar density, which represent the main source of systematic errors, $P_{\text {meas,nw }}(k)$ is the measured power spectrum without these weights and $S$ is an extra nuisance parameter to be marginalized over, see Table III. The expectation value of the matter power spectrum is a convolution of the true matter power spectrum with the window functions, which account for the correlation of data at different scales $k$ due to the survey geometry. Therefore, the theoretical power spectra $P_{\mathrm{th}}^{g}(k)$ (to be computed in the following section) needs to be convolved with 
TABLE III. Uniform priors for the DR9 bias and shot noise parameters $b_{\mathrm{HF}}$ and $P_{\mathrm{HF}}^{\mathrm{s}}$ respectively, in the case of the HaloFit prescription for the galaxy power spectrum as well as for $b_{\mathrm{Q}}$ and $Q$, free parameters of the model of Ref. [43]. We explore the neutrino mass constraints for these two galaxy clustering models in the case of the DR9 3D power spectrum.

\begin{tabular}{lc}
\hline \hline DR9 parameters & Prior \\
\hline$S$ & $-1 \rightarrow 1$ \\
$b_{\mathrm{HF}}$ & $0.1 \rightarrow 10$ \\
$P_{\mathrm{HF}}^{\mathrm{s}}$ & $100 \rightarrow 10000$ \\
$b_{\mathrm{Q}}$ & $0 . \rightarrow 10$ \\
$Q$ & $0.1 \rightarrow 100$ \\
\hline \hline
\end{tabular}

a window matrix before comparing it to $P_{\text {meas }}(k)$. In order to avoid nonlinearities, we adopt the conservative choice of a maximum wave number of $k_{\max }=0.12 \mathrm{~h} / \mathrm{Mpc}$, a region which is safe against large nonlinear corrections in the modeled theoretical spectra, which we discuss below. We use therefore 22 points in the range $0.03 h / \mathrm{Mpc}<k<$ $0.12 \mathrm{~h} / \mathrm{Mpc}$ from the total 74 points of the DR9 power spectrum.

\section{DR9 clustering model}

We follow here two different approaches to model the theoretical power spectrum in the weakly nonlinear regime explored here $\left(k_{\max }=0.12 \mathrm{~h} / \mathrm{Mpc}\right)$. These two models are among the three considered in Ref. [9], where it was checked that the neutrino mass bounds show a very mild dependence on the galaxy clustering models considered in their analyses. The first approach we consider for DR9 is the HaloFit prescription (HF) [31,32]. In Ref. [44] an extension to HaloFit that accounts for the effect of massive neutrinos in the nonlinear regime has been developed. However, we do not follow this prescription as the corrections induced to the standard HaloFit are completely negligible at the scales and redshifts of interest here, in agreement with the findings of Refs. [45,46].

The final theoretical galaxy power spectrum to be convolved with the window functions reads

$$
P_{\mathrm{th}}^{g}(k, z)=b_{\mathrm{HF}}^{2} P_{\mathrm{HF} \nu}^{\mathrm{m}}(k ; z)+P_{\mathrm{HF}}^{\mathrm{s}},
$$

where $b_{\mathrm{HF}}$ and $P_{\mathrm{HF}}^{\mathrm{s}}$ are the bias and the shot contribution respectively, considered to be constant. The priors adopted in the the former two parameters are depicted in Table III. The model given above by Eq. (7) with a bias and a shot noise parameter is equivalent to that used before for modeling the theoretical angular power spectra of DR8 data analyses; see Eq. (2).

The second approach adopted here for galaxy clustering modeling is that of Ref. [43]:

$$
P_{\mathrm{th}}^{g}(k, z)=b_{\mathrm{Q}}^{2} \frac{1+Q k^{2}}{1+1.4 k} P^{\mathrm{m}, \text { linear }}(k, z),
$$

where $k$ is the wave number in units of $\mathrm{h} / \mathrm{Mpc}$ and $P^{\mathrm{m} \text {,linear }}$ is the linear matter power spectrum. The free parameters of this model are $b_{\mathrm{Q}}$ and $Q$, which mimic the scale dependence of the power spectrum at small scales. These two parameters are considered here constants with priors specified in Table III. In the following section we shall comment on the dependence of the neutrino mass constraints on the underlying galaxy power spectrum model.

\section{RESULTS}

Here we present the constraints from current cosmological data sets on the sum of the three active neutrino masses $\sum m_{\nu}$ in different scenarios and with different combinations of data sets.

\section{A. Standard cosmology plus massive neutrinos}

Throughout this section we shall assume a $\Lambda \mathrm{CDM}$ cosmology, and compute the bounds on the sum of the three active neutrino masses arising from the different cosmological data sets considered here. Table IV shows the 95\% C.L. upper bounds on the total neutrino mass for PLANCK, PLANCK plus DR8 and PLANCK plus DR9 data sets, with and without the HST prior on the Hubble constant. These limits include the shot noise additional parameters in the case of DR8 and the systematic effects, in the case of DR9. Notice first that the constraints from the PLANCK data set described before (which include the Planck lensing likelihood as well as WMAP 9-year data polarization measurements) are not very promising, since in this case $\sum m_{\nu}<1.11 \mathrm{eV}$ at $95 \%$ C.L. The fact that $\mathrm{CMB}$ alone does not provide very significant constraints on the sum of the neutrino masses has been already discussed in the literature (see, for instance [8]). Indeed, without the $H_{0}$ prior, the change induced in the CMB temperature anisotropies caused by an increase in $\sum m_{\nu}$ can be compensated by a decrease in the Hubble constant $H_{0}$. An increase in $\sum m_{\nu}$ will induce a shift in the distance to last scattering. ${ }^{2}$ While the acoustic peak structure of the CMB data does not leave much freedom in $\omega_{c}$ and $\omega_{b}$, the change in distance to last scattering could be compensated by lowering $H_{0}$. The presence of the HST prior on the Hubble parameter will break this strong degeneracy, setting a 95\% C.L. bound of $0.22 \mathrm{eV}$ in the sum of the three active neutrino masses.

However, and as discussed in the introductory section, HST and Planck data sets show a tension of $\sim 2.5 \sigma$ in their measured value of the Hubble constant $H_{0}$. It is therefore mandatory to explore whether other data sets could also strengthen the constraint on $\sum m_{\nu}$ from the PLANCK data set alone. DR8 angular power spectrum measurements, if combined with the PLANCK data set, provide an upper

\footnotetext{
${ }^{2} r_{\theta}\left(z_{\mathrm{rec}}\right) \propto \int_{0}^{z_{\mathrm{rec}}} d z\left[\omega_{r} a^{-4}+\omega_{m} a^{-3}+\left(1-\omega_{m} / h^{2}\right)\right]^{-1 / 2}$, with $\omega_{m}=\omega_{b}+\omega_{c}+\omega_{\nu}$.
} 
TABLE IV. $\quad 95 \%$ C.L. upper bounds on $\Sigma m_{\nu}$ in a $\Lambda$ CDM model from the different data combinations considered here, with (without) the HST prior on the Hubble constant $H_{0}$. The results with DR8 (DR9) data sets include the shot noise (the systematic corrections) parameters.

\begin{tabular}{cccc}
\hline \hline & Planck + WP + lensing $(+$ HST $)$ & Planck + WP + lensing + DR8 $(+$ HST $)$ & Planck + WP + lensing + DR9(+HST) \\
\hline$\sum m_{\nu}[\mathrm{eV}]$ & $<1.11(0.22)$ & $<0.98(0.23)$ & $<0.39(0.23)$ \\
\hline \hline
\end{tabular}

limit of $\sum m_{\nu}<0.98 \mathrm{eV}$ at $95 \%$ C.L. with the shot noise parameters included in the analysis. If we consider instead the DR8 BAO angular diameter distance constraint $D_{A}(z)=$ $1411 \pm 65 \mathrm{Mpc}$ at $z=0.54$ [30] and combine this measurement with the PLANCK data set, the bound is $\sum m_{\nu}<$ $0.85 \mathrm{eV}$ at $95 \%$ C.L. The neutrino mass bound from DR8 BAO only is mildly stronger than the one obtained with the full shape of the DR8 galaxy clustering matter spectrum due to the larger value of $\ell_{\max }=300$ used in the analysis of Ref. [30] to extract the angular BAO signature.

When considering the DR9 data set combined with PLANCK, we achieve a bound of $\sum m_{\nu}<0.39 \mathrm{eV}$ at $95 \%$ C.L. The former limit is obtained in the case in which the theoretical power spectrum for DR9 is given by Eq. (7) which uses the HF prescription. Very similar bounds are obtained if we use for the theoretical DR9 spectrum the approach given by Eq. (8).

If instead of using the full shape information from BOSS DR9 we use the DR9 BAO signature [19], the neutrino mass limit is $\sum m_{\nu}<0.40 \mathrm{eV}$ at $95 \%$ C.L. Note that the bound on $\sum m_{\nu}$ arising from the geometrical BAO DR9 geometrical information is very similar to that obtained using the full shape of the DR9 3D clustering measurements. While in the context of the minimal $\Lambda \mathrm{CDM}$ model, BAO measurements and Galaxy clustering data should provide similar constraints, the BAO DR9 signal is extracted using the matter power spectrum in the range $0.02 h / \mathrm{Mpc}<k<0.3 h / \mathrm{Mpc}$ [19], a much wider range than the one considered in the full power spectrum case.

To summarize, Galaxy clustering data, and, especially, DR9 3D power spectrum data, helps enormously in improving the neutrino mass constraints, arriving at $m_{\nu}<$ $0.39 \mathrm{eV}$ at $95 \%$ C.L. without the addition of the measurement of $H_{0}$ from the HST experiment. The former bound is not as tight as the value quoted by the Planck collaboration $\sum m_{\nu}<0.26 \mathrm{eV}$ at $95 \%$ C.L., obtained after combining Planck measurements (including lensing) with WP and $\mathrm{BAO}$ data. The reason for the difference among these two $95 \%$ C.L. neutrino mass bounds (i.e. $\sum m_{\nu}<$ $0.39 \mathrm{eV}$ versus $\sum m_{\nu}<0.26 \mathrm{eV}$ ) is due to the fact that here we are considering exclusively BAO information from DR9 SDSS data, while in the Planck analysis other available BAO measurements have been considered as well.

\section{B. Dark energy and massive neutrinos}

In this section we explore the bounds on the sum of neutrino masses if the dark energy equation of state $w$ is allowed to vary ( $w$ CDM model). There exists a strong and very well-known degeneracy in the $\sum m_{\nu}-w$ plane [47]. If the neutrino mass is allowed to freely vary, the amount of cold dark matter is required to increase in order to leave the matter power spectrum unchanged. This change of $\Omega_{m}$ can also occur if $w$ is allowed to freely vary as well. Consequently, cosmological neutrino mass bounds will become weaker if the dark energy equation of state is included as a free parameter. Table $\mathrm{V}$ presents the galaxy clustering limits on the sum of neutrino masses and on the dark energy equation of state $w$ within the $w$ CDM scenario. For the sake of comparison, we depict as well the constraints from the PLANCK data set alone. The addition of HST data to the basic PLANCK CMB data set barely changes the 95\% C.L. constraint of $\sum m_{\nu}<1 \mathrm{eV}$. While the addition of DR8 BOSS data, neither in the form of clustering measurements nor in the form of geometrical BAO constraints, changes these limits significantly, ${ }^{3}$ the addition of the DR9 3D power spectrum measurements sets a 95\% C.L. limit of $\sum m_{\nu}<0.48 \mathrm{eV}$. This limit is much better than the one provided by the combination of DR9 BAO information [19] and the PLANCK data set in a $w C D M$ universe, which is $\sum m_{\nu}<0.71 \mathrm{eV}$ at 95\% C.L.

Concerning $w$, the mean values and the $95 \%$ C.L. associated errors depicted in Table V show that the combination of galaxy clustering measurements with the PLANCK CMB data set is not able to extract $w$ with high precision: the constraints we obtained from this data combination for $w$ are rather weak but perfectly consistent with a $\Lambda \mathrm{CDM}$ model. The addition of Supernovae Ia luminosity distance measurements from the 3-year Supernova Legacy Survey (SNLS3) [48] reduces significantly the errors on the dark energy equation of state: the combination of PLANCK plus SNLS3 provides a mean value and $95 \%$ C.L. errors on the dark energy equation of state parameter of $w=$ $-1.21_{-0.22}^{+0.20}$. If DR9 Galaxy clustering data is also added in the analysis, $w=-1.16_{-0.17}^{+0.15}$.

Figure 1, left panel, shows the $68 \%$ and $95 \%$ C.L. allowed regions in the $\left(\sum m_{\nu}, w\right)$ plane from the PLANCK data set described in Sec. III, and also from the combination of the former data set with DR9 BAO geometrical information and with DR9 galaxy clustering

\footnotetext{
${ }^{3}$ Without shot noise parameters the addition of DR8 angular power spectrum to Planck data results in a much better constraint than the one quoted in Table $\mathrm{V}$, being $\sum m_{\nu}<0.77 \mathrm{eV}$ at $95 \%$ C.L.
} 
TABLE V. $95 \%$ C.L. upper bounds on $\Sigma m_{\nu}$ from the different data combinations considered here within a $w$ CDM model, with (without) the HST prior on the Hubble constant $H_{0}$. We show as well the mean value of $w$ together with its $95 \%$ C.L. errors. The results with DR8 (DR9) data sets refer to the case in which the full shape of the angular (3D) power spectrum is considered, including shot noise parameters (systematic corrections) in the analyses. The constraint from the full shape of DR9 galaxy clustering measurements is highly superior to that arising from the combination of DR9 BAO information [19] and the PLANCK data set in a $w C D M$ universe, which is $\sum m_{\nu}<0.71 \mathrm{eV}$ at $95 \%$ C.L.

\begin{tabular}{lccc}
\hline \hline & Planck + WP + lensing(+HST) & Planck + WP + lensing + DR8 (+HST) & Planck + WP + lensing + DR9(+HST) \\
\hline$\sum m_{\nu}[\mathrm{eV}]$ & $<1.01(0.97)$ & $<1.02(0.95)$ & $<0.48(0.58)$ \\
$w$ & $-1.55_{-0.45}^{+0.54}$ & $-1.42_{-0.58}^{+0.49}$ & $-1.10_{-0.57}^{+0.44}$ \\
& $\left(-1.53_{-0.45}^{+0.37}\right)$ & $\left(-1.54_{-0.37}^{-0.45}\right)$ & $\left(-1.30_{-0.34}^{+0.30}\right)$ \\
\hline \hline
\end{tabular}

(i.e. full shape) measurements. Notice that the neutrino mass limits using the galaxy clustering information are better than those obtained using the BAO signature alone. Indeed, DR9 BAO measurements show a mild preference for $w<-1$, allowing therefore for a larger neutrino mass. We also investigate the impact of adding Supernovae Ia luminosity distance constraints to the combination of PLANCK and DR9 galaxy clustering data sets: while the impact on the sum of the neutrino mass bound is negligible, the errors on the dark energy equation of state parameter $w$ are reduced by a factor of 3 .

\section{Curvature and massive neutrinos}

We present here the constraints on neutrino masses in the context of a nonflat universe, allowing for a nonnegligible curvature component; see Table I for the priors adopted in the curvature component. Table VI shows our constraints for the PLANCK data set, PLANCK plus DR8 angular power spectrum data and PLANCK plus DR9 galaxy clustering measurements with and without a prior on the Hubble constant $H_{0}$ from HST. In this nonflat model, DR8 angular clustering measurements combined with PLANCK reduce the constraint on $\sum m_{\nu}$, from

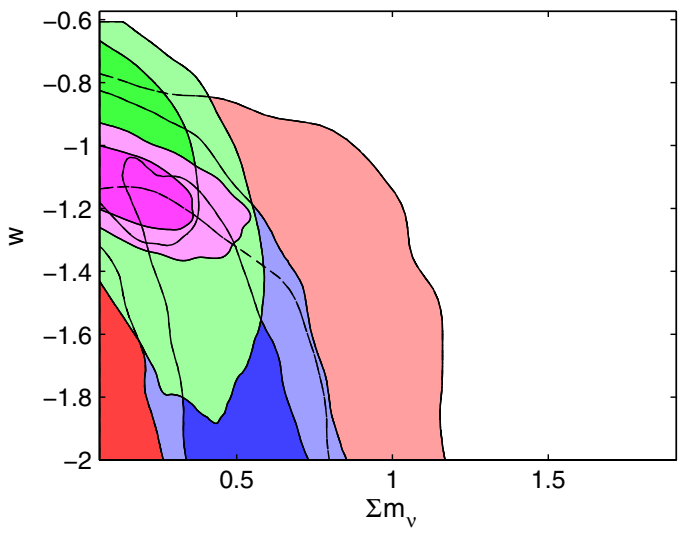

$\sum m_{\nu}<1.36 \mathrm{eV}$ to $\sum m_{\nu}<0.92 \mathrm{eV}$ (both at $95 \%$ C.L.). This constraint is very similar to the one obtained if the BAO DR8 geometrical information is used, $\sum m_{\nu}<$ $0.80 \mathrm{eV}$. Adding the HST prior to DR8 angular power spectrum measurements improves significantly the constraints: the $95 \%$ C.L. upper limit is $\sum m_{\nu}<0.33 \mathrm{eV}$.

DR9 3D power spectrum measurements greatly improve the results from the PLANCK data set: when combined with our basic PLANCK data set, the 95\% C.L. bounds without the HST prior are $\sum m_{\nu}<0.35 \mathrm{eV}$ with systematic uncertainties. If HST data is included as well in the analysis, the former $95 \%$ C.L. bound translates into $\sum m_{\nu}<0.26 \mathrm{eV}$. These limits are better than those obtained from the combination of the PLANCK data set with the DR9 BAO measurement, which is $\sum m_{\nu}<$ $0.47 \mathrm{eV}$ without the HST prior. Therefore, this nonflat model, together with the $w C D M$ one, is a working example in which constraints from full shape 3D power-spectrum measurements provide significant extra information than those from BAO signature alone.

Figure 1, right panel, shows the $68 \%$ and 95\% C.L. allowed regions in the $\left(\sum m_{\nu}, \Omega_{k}\right)$ plane from the PLANCK data set described in Sec. III, and from the

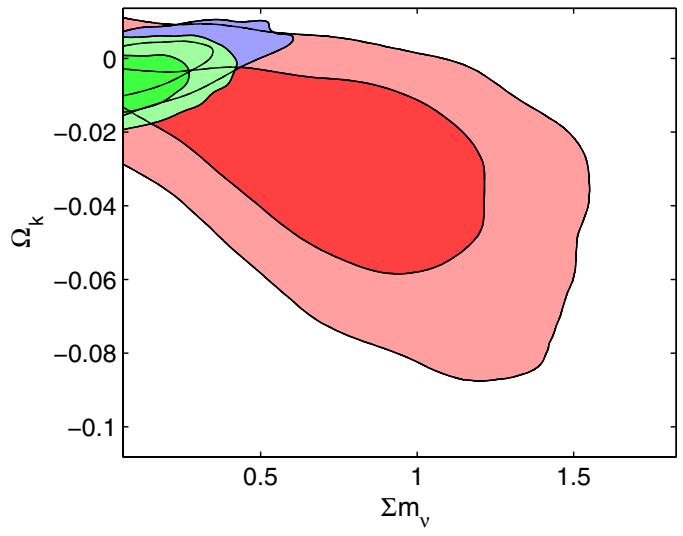

FIG. 1 (color online). Left panel: The red (large) contours show the 68\% and 95\% C.L. allowed regions from the PLANCK data set in the $\left(\sum m_{\nu}, w\right)$ plane, while the blue (thinner, lower contours) and green (thinner, upper) contours show the impact of the addition of the DR9 BAO signature and the full shape of DR9 galaxy clustering measurements, respectively. The small magenta contours depict the combination of PLANCK with DR9 Galaxy clustering data and SNLS3 measurements. Right panel: The red (large) contours show the $68 \%$ and $95 \%$ C.L. allowed regions from the PLANCK data set, while the blue (thinner, upper contours) and green (thinner, lower) contours show the impact of the addition of the DR9 BAO signature and the full shape of DR9 galaxy clustering measurements, respectively. Note the absence of the case with SNLS3 data in the analyses presented in this figure. 
TABLE VI. $\quad 95 \%$ C.L. upper bounds on $\Sigma m_{\nu}$ in a nonflat model from the different data combinations considered here, with (without) the HST prior on the Hubble constant $H_{0}$. We depict as well the mean value and the $95 \%$ C.L. errors for the curvature energy density $\Omega_{k}$. The results with DR8 (DR9) data sets refer to the case in which the full shape of the angular (3D) power spectrum is considered, including shot noise parameters (systematic corrections) in the analyses. The neutrino mass bound extracted from the full shape measurements of BOSS DR9 are better than the one obtained using the DR9 BAO measurement [19], which is $\sum m_{\nu}<0.47 \mathrm{eV}$ at 95\% C.L. without the HST prior.

\begin{tabular}{lccc}
\hline \hline & Planck + WP + lensing $(+$ HST $)$ & Planck + WP + lensing + DR8 $(+$ HST $)$ & Planck + WP + lensing + DR9 $(+$ HST $)$ \\
\hline$\sum m_{\nu}[\mathrm{eV}]$ & $<1.36(0.32)$ & $<0.92(0.33)$ & $<0.35(0.26)$ \\
$\Omega_{\mathrm{k}}$ & $-0.031_{-0.041}^{+0.036}$ & $-0.01_{-0.019}^{+0.018}$ & $0.005_{-0.009}^{+0.01}$ \\
& $0.007_{-0.010}^{+0.009}$ & $\left(0.006_{-0.010}^{+0.010}\right)$ & $\left(0.001_{-0.009}^{+0.008}\right)$ \\
\hline \hline
\end{tabular}

combination of the former data set with DR9 BAO measurements, and DR9 galaxy clustering information. Notice that the neutrino mass constraint arising from the clustering measurements is more powerful than those obtained exploiting the $\mathrm{BAO}$ signature.

Concerning $\Omega_{k}$, the mean value and the associated 95\% C.L. errors are not significantly changed when galaxy clustering measurements are included.

\section{CONCLUSIONS}

Cosmology provides an independent laboratory to test physical properties of fundamental particles. Neutrino masses affect the different cosmological observables in different ways, and therefore it is possible to derive strong constraints on the sum of their masses by combining different cosmological data sets. Cosmic microwave background physics is affected by the presence of massive neutrinos via the Early Integrated Sachs Wolfe effect, since the transition from the relativistic to the nonrelativistic neutrino regime will induce a nontrivial evolution of the metric perturbations. Massive neutrinos will also suppress the lensing potential.

Large scale structure measurements of the galaxy power spectrum are affected by massive neutrinos, since they are hot relics with large velocity dispersion which, at a given redshift, erase the growth of matter perturbations on spatial scales smaller than the typical neutrino free streaming scale. Recent measurements of the Planck CMB experiment do not provide a strong bound on the sum of the neutrino masses. The addition of a prior on the Hubble constant from the Hubble Space Telescope improves the results in a very significant way since it breaks the strong degeneracy between the neutrino mass and the Hubble constant. However, Planck and HST data sets show some tension in the measurement of the Hubble parameter. While baryon acoustic oscillation measurements also improve the neutrino mass bounds when combined with Planck data, it is crucial to explore if measurements using the full shape of the matter power spectrum can further improve the neutrino mass limits, in particular, in nonminimal cosmological scenarios with a curvature or with a dark energy equation of state $w \neq-1$.
Here we combine recent Planck data with galaxy power spectrum measurements from the BOSS experiment, one of the four surveys of the Sloan Digital Sky Survey III, (SDSS-III) to derive the constraints on the sum of neutrino masses. We explore both the 2D angular power spectrum measurements from the CMASS sample of luminous galaxies of SDSS-III Data Release 8 (DR8) as well as the full 3D power spectrum shape of SDSS-III Data Release 9 (DR9).

In the context of a minimal $\Lambda \mathrm{CDM}$ scenario, DR9 3D galaxy clustering measurements improve significantly the existing neutrino mass bounds from Planck data. We find $\sum m_{\nu}<0.39 \mathrm{eV}$ at $95 \%$ confidence level for the combination of the DR9 3D power spectrum with Planck CMB data (with lensing included) and Wilkinson Microwave Anisoptropy Probe 9-year polarization measurements. Similar results are obtained with the DR9 BAO geometrical signature. Therefore, the $95 \%$ confidence level constraint of $\sum m_{\nu}<1.1 \mathrm{eV}$ obtained in the absence of large scale structure measurements is greatly improved and robust neutrino mass constraints can be obtained without the addition of the controversial prior on the Hubble constant from HST.

In the presence of a freely varying (constant) dark energy equation of state, we find $\sum m_{\nu}<0.49 \mathrm{eV}$ at 95\% confidence level for the combination of the DR9 3D power spectrum with Planck CMB data (with lensing included) and Wilkinson Microwave Anisoptropy Probe 9-year polarization measurements, making this constraint highly superior to that obtained when replacing Galaxy clustering data by the HST prior.

In nonflat geometries, the combination of the DR9 3D power spectrum with Planck CMB data (with lensing included) and Wilkinson Microwave Anisoptropy Probe 9-year polarization measurements provides the neutrino mass bound $\sum m_{\nu}<0.35 \mathrm{eV}$ at $95 \%$ confidence level. If we use instead the associated DR9 BAO geometrical info, the $95 \%$ confidence level neutrino mass bounds in the ${ }_{w C D M}$ and nonflat cosmologies are $\sum m_{\nu}<0.71 \mathrm{eV}$ and $\sum m_{\nu}<0.46 \mathrm{eV}$, respectively. Consequently, in extended cosmological scenarios with a free dark energy equation of state or with a curvature component, measurements of the full shape of the galaxy power spectrum are 
extremely helpful, providing better results than those obtained with the associated baryon acoustic oscillation signature only.

While we were completing this study, a new analysis [49] combining Planck data, galaxy clustering measurements from the WiggleZ Dark Energy Survey and other external data sets has appeared in the literature. The bound is $\sum m_{\nu}<0.24 \mathrm{eV}$ at $95 \%$ confidence level when combining Planck with WiggleZ power spectrum measurements, setting $k_{\max }=0.2 \mathrm{~h} / \mathrm{Mpc}$. The analyses presented here are however penalized by our large systematic uncertainties: as a comparison, when we neglect in our analyses systematic uncertainties, we get $\sum m_{\nu}<0.25 \mathrm{eV}$ at $95 \%$ confidence level after combining Planck with DR9 galaxy clustering measurements (with a shot noise nuisance parameter included and setting $\left.k_{\max }=0.12 h / \mathrm{Mpc}\right)$.

\section{ACKNOWLEDGMENTS}

The authors would like to thank Shun Saito for help concerning DR9 power spectrum measurements and Maria Archidiacono for useful help with the manuscript. Part of the research described in this paper was carried out at the Jet Propulsion Laboratory, California Institute of Technology, under a contract with the National Aeronautics and Space Administration. O. M. is supported by the Consolider Ingenio Project No. CSD2007-00060, by PROMETEO/2009/116, by the Spanish Ministry Science Project No. FPA2011-29678 and by the ITN Invisibles PITN-GA-2011-289442.
[1] J. Lesgourgues and S. Pastor, Adv. High Energy Phys. 2012, 608515 (2012).

[2] P.A.R. Ade et al. (Planck Collaboration), arXiv:1303.5076.

[3] J. Lesgourgues, L. Perotto, S. Pastor, and M. Piat, Phys. Rev. D 73, 045021 (2006).

[4] J. Lesgourgues and S. Pastor, Phys. Rep. 429, 307 (2006).

[5] B. A. Reid, L. Verde, R. Jimenez, and O. Mena, J. Cosmol. Astropart. Phys. 01 (2010) 003.

[6] J. Hamann, S. Hannestad, J. Lesgourgues, C. Rampf, and Y. Y. Y. Wong, J. Cosmol. Astropart. Phys. 07 (2010) 022.

[7] R. de Putter, O. Mena, E. Giusarma, S. Ho, A. Cuesta, H.-J. Seo, A. Ross, M. White et al., Astrophys. J. 761, 12 (2012).

[8] E. Giusarma, R. de Putter, and O. Mena, Phys. Rev. D 87, 043515 (2013).

[9] G.-B. Zhao, S. Saito, W. J. Percival, A. J. Ross, F. Montesano, M. Viel, D. P. Schneider, M. Manera et al., arXiv:1211.3741.

[10] G. Hinshaw, D. Larson, E. Komatsu, D. N. Spergel, C. L. Bennett, J. Dunkley, M. R. Nolta, M. Halpern et al., arXiv:1212.5226.

[11] Z. Hou, C. L. Reichardt, K. T. Story, B. Follin, R. Keisler, K. A. Aird, B. A. Benson, L.E. Bleem et al., arXiv:1212.6267.

[12] J. L. Sievers, R. A. Hlozek, M. R. Nolta, V. Acquaviva, G. E. Addison, P. A. R. Ade, P. Aguirre, M. Amiri et al., arXiv:1301.0824.

[13] M. Archidiacono, E. Giusarma, A. Melchiorri, and O. Mena, Phys. Rev. D 87, 103519 (2013).

[14] C. L. Bennett, D. Larson, J.L. Weiland, N. Jarosik, G. Hinshaw, N. Odegard, K. M. Smith, R. S. Hill et al., arXiv: 1212.5225 .

[15] A. G. Riess, L. Macri, S. Casertano, H. Lampeitl, H. C. Ferguson, A. V. Filippenko, S. W. Jha, W. Li, and R. Chornock, Astrophys. J. 730, 119 (2011); A. G. Riess, L. Macri, S. Casertano, H. Lampeit, H.C. Ferguson, A. V. Filippenko, S. W. Jha, W. Li, R. Chornock, and
J. M. Silverman, Astrophys. J. 732, 129(E) (2011); arXiv:1103.2976.

[16] W. J. Percival et al. (SDSS Collaboration), Mon. Not. R. Astron. Soc. 401, 2148 (2010).

[17] N. Padmanabhan, X. Xu, D. J. Eisenstein, R. Scalzo, A. J. Cuesta, K. T. Mehta, and E. Kazin, Mon. Not. R. Astron. Soc. 427, 2132 (2012).

[18] C. Blake, E. Kazin, F. Beutler, T. Davis, D. Parkinson, S. Brough, M. Colless, C. Contreras et al., Mon. Not. R. Astron. Soc. 418, 1707 (2011).

[19] L. Anderson, E. Aubourg, S. Bailey, D. Bizyaev, M. Blanton, A. S. Bolton, J. Brinkmann, J. R. Brownstein et al., Mon. Not. R. Astron. Soc. 427, 3435 (2013).

[20] F. Beutler, C. Blake, M. Colless, D. H. Jones, L. StaveleySmith, L. Campbell, Q. Parker, W. Saunders, and F. Watson, Mon. Not. R. Astron. Soc. 416, 3017 (2011).

[21] K. S. Dawson et al. (BOSS Collaboration), arXiv:1208.0022.

[22] D. J. Eisenstein et al. (SDSS Collaboration), Astron. J. 142, 72 (2011).

[23] S. Ho, A. Cuesta, H.-J. Seo, R. de Putter, A. J. Ross, M. White, N. Padmanabhan, S. Saito et al., Astrophys. J. 761, 14 (2012).

[24] M. White, M. Blanton, A. Bolton, D. Schlegel, J. Tinker, A. Berlind, L. da Costa, E. Kazin et al., Astrophys. J. 728, 126 (2011).

[25] H. Aihara et al. (SDSS Collaboration), Astrophys. J. Suppl. Ser. 193, 29 (2011); 195, 26(E) (2011); arXiv:1101.1559.

[26] C. P. Ahn et al. (SDSS Collaboration), Astrophys. J. Suppl. Ser. 203, 21 (2012).

[27] A. Lewis, A. Challinor, and A. Lasenby, Astrophys. J. 538, 473 (2000).

[28] A. Lewis and S. Bridle, Phys. Rev. D 66, 103511 (2002).

[29] A. J. Ross, S. Ho, A. J. Cuesta, R. Tojeiro, W. J. Percival, D. Wake, K. L. Masters, R. C. Nichol et al., Mon. Not. R. Astron. Soc. 417, 1350 (2011).

[30] H.-J. Seo, S. Ho, M. White, A. Cuesta, A. Ross, S. Saito, B. Reid, N. Padmanabhan et al., Astrophys. J. 761, 13 (2012). 
[31] R.E. Smith, J. A. Peacock, A. Jenkins, S. D. M. White, C. S. Frenk, F. R. Pearce, P. A. Thomas, G. Efstathiou, and H. M.P. Couchman (Virgo Consortium Collaboration), Mon. Not. R. Astron. Soc. 341, 1311 (2003).

[32] R. Takahashi, M. Sato, T. Nishimichi, A. Taruya, and M. Oguri, Astrophys. J. 761, 152 (2012).

[33] R. E. Smith, J. A. Peacock, A. Jenkins, S. D. M. White, C. S. Frenk, F. R. Pearce, P. A. Thomas, G. Efstathiou, and H. M.P. Couchman (Virgo Consortium Collaboration), Mon. Not. R. Astron. Soc. 341, 1311 (2003).

[34] R. A. Vanderveld, M. J. Mortonson, W. Hu, and T. Eifler, Phys. Rev. D 85, 103518 (2012).

[35] B. A. Reid, L. Samushia, M. White, W. J. Percival, M. Manera, N. Padmanabhan, A. J. Ross, A. G. Sanchez et al., arXiv:1203.6641.

[36] L. Samushia, B. A. Reid, M. White, W. J. Percival, A. J. Cuesta, L. Lombriser, M. Manera, R. C. Nichol et al., Mon. Not. R. Astron. Soc. 429, 1514 (2013).

[37] A. G. Sanchez, C. G. Scoccola, A. J. Ross, W. Percival, M. Manera, F. Montesano, X. Mazzalay, A. J. Cuesta et al., arXiv:1203.6616.

[38] S. E. Nuza, A. G. Sanchez, F. Prada, A. Klypin, D. J. Schlegel, S. Gottloeber, A. D. Montero-Dorta, M. Manera et al., arXiv:1202.6057.
[39] A. J. Ross et al. (BOSS Collaboration), Mon. Not. R. Astron. Soc. 424, 564 (2012).

[40] A. J. Ross, W. J. Percival, A. Carnero, G.-b. Zhao, M. Manera, A. Raccanelli, E. Aubourg, D. Bizyaev et al., arXiv:1208.1491.

[41] H. A. Feldman, N. Kaiser, and J. A. Peacock, Astrophys. J. 426, 23 (1994).

[42] B. A. Reid, W. J. Percival, D. J. Eisenstein, L. Verde, D. N. Spergel, R. A. Skibba, N. A. Bahcall, T. Budavari et al., Mon. Not. R. Astron. Soc. 404, 60 (2010).

[43] S. Cole et al. (2dFGRS Collaboration), Mon. Not. R. Astron. Soc. 362, 505 (2005).

[44] S. Bird, M. Viel, and M. G. Haehnelt, Mon. Not. R. Astron. Soc. 420, 2551 (2012).

[45] S. Saito, M. Takada, and A. Taruya, Phys. Rev. D 80, 083528 (2009).

[46] S. Hannestad, T. Haugbolle, and C. Schultz, J. Cosmol. Astropart. Phys. 02 (2012) 045.

[47] S. Hannestad, Phys. Rev. Lett. 95, 221301 (2005).

[48] A. Conley, J. Guy, M. Sullivan, N. Regnault, P. Astier, C. Balland, S. Basa, R. G. Carlberg et al., Astrophys. J. Suppl. Ser. 192, 1 (2011).

[49] S. Riemer-Srensen, D. Parkinson, and T.M. Davis, arXiv:1306.4153. 